\title{
Dual control Monte-Carlo method for tight bounds of value function in regime switching utility maximization
}

\author{
Jingtang Ma*, Wenyuan Li†and Harry Zheng ‡
}

\begin{abstract}
In this paper we study the dual control approach for the optimal asset allocation problem in a continuous-time regime-switching market. We find the lower and upper bounds of the value function that is a solution to a system of fully coupled nonlinear partial differential equations. These bounds can be tightened with additional controls to the dual process. We suggest a Monte-Carlo algorithm for computing the tight lower and upper bounds and show the method is effective with a variety of utility functions, including power, non-HARA and Yaari utilities. The latter two utilities are beyond the scope of any current methods available in finding the value function.
\end{abstract}

MSC 2010: 49L20, 90C46, 65C05

Keywords: Portfolio optimization, regime switching, dual control, non-HARA utility, Yaari utility, tight lower and upper bounds, Monte-Carlo method

\section{Introduction}

Stochastic control methods are widely used in solving dynamic portfolio optimization problems in finance, among many other applications. The key idea is to apply the dynamic programming principle to the optimal value function and show that it satisfies a partial differential equation (PDE), called the Hamilton-Jacobi-Bellman (HJB) equation. If one can find a classical solution to the HJB equation, then one may verify it is the value function by the martingale principle of optimality, and find a feedback optimal control as a by-product. Otherwise, one may show the value function is a unique viscosity solution to the HJB equation and find it numerically, see the excellent exposition of stochastic control and its applications in Pham (2009).

Since the HJB equation is a fully nonlinear PDE, its solvability crucially depends on the terminal condition. In a Black-Scholes complete market model with a power or logarithmic utility, we know there is a closed-form classical solution to the HJB equation. Bian et al.

\footnotetext{
*School of Economic Mathematics and Collaborative Innovation Center of Financial Security, Southwestern University of Finance and Economics, Chengdu, 611130, China (Email: mjt@swufe.edu.cn). The work was supported by National Natural Science Foundation of China (Grant No. 11671323) and Program for New Century Excellent Talents in University (China Grant No. NCET-12-0922).

${ }^{\dagger}$ School of Economic Mathematics, Southwestern University of Finance and Economics, Chengdu, 611130, China (Email: Wylfm@2011.swufe.edu.cn).

${ }^{\ddagger}$ Corresponding author. Department of Mathematics, Imperial College, London SW7 2BZ, UK (Email: h.zheng@imperial.ac.uk).
} 
(2011) and Bian and Zheng (2015) use the dual control method to show there is a classical solution to the HJB equation for a broad class of utility functions and give a representation of the solution to the HJB equation in terms of that of the dual HJB equation. For constrained market models one may have to use numerical method to solve the HJB equation, see, for example, Huang et al. (2012) for a combined fixed point and policy iterations method and Reisinger and Forsyth (2016) for a piecewise constant policy approximations method. In this paper we discuss the method of finding the approximate solution to the HJB equation arising from regime switching (RS) utility maximization problems.

The RS model is popular in financial data modelling and analysis as it allows parameters of asset price dynamics to depend on a finite state Markov chain process (MCP). It has been shown in the literature that MCP is effective in providing information of the market environment. For example, Hamilton (1989) introduces a RS model for nonstationary time series and business cycles. Hardy (2001) applies a two-regime model to provide a good fit to monthly stock market returns. The RS model provides good flexibility for characterizing macro market uncertainties while preserves analytic tractability for underlying asset price dynamics. There has been active research in portfolio optimization with RS models. For example, Zhang et al. (2005) and Yin et al. (2006) study the trading rules in a RS market. Zhou and Yin (2003) and Çelikyurt and Özekici (2007) investigate the mean-variance portfolio optimization in a discrete-time RS model. Çanakoğlu and Özekici (2012) discuss the HARA utility maximization in a continuous-time RS model. Honda (2003), Sass and Haussmann (2004), and Rieder and Bäuerle (2005) solve portfolio optimization problems with partial information and regime-switching drift processes.

For a regime switching stochastic control problem, Bäuerle and Rieder (2004) and Fu et al. (2014) show that the value function satisfies the HJB equation which is a system of fully coupled nonlinear PDEs and prove a verification theorem. Furthermore, for a power or logarithmic utility function, they reduce the HJB equation to a system of linear ODEs which are then solved with matrix exponentials. For general utility functions, it seems highly unlikely, if not impossible, that one can solve the system of HJB equations analytically. There are some great efforts in the literature in designing numerical schemes. Huang et al. (2011) discuss numerical methods for pricing American options under regime switching and use various policy iterations to find the solution of the discretized HJB variational equations and show the convergence of iterations under some strong technical assumptions. It is difficult to apply the methods of Huang et al. (2011) to RS utility maximization as at each grid point one needs to solve an optimization problem over some unknown value function, which would result in a sequence of systems of highly nonlinear algebraic equations and would be challenging and time-consuming to solve them. Similarly, it is not clear how one may design a monotone scheme for discretizing the system of HJB equations and analyzing the consistency and stability with the techniques in Forsyth and Labahn (2008) for a single HJB equation. Fu et al. (2014) introduce a functional operator to generate a sequence of value functions and show that the optimal value function is the limit of this sequence. Each function in the sequence solves an auxiliary portfolio optimization problem in a single-regime market and is a solution to the HJB equation. The method in Fu et al. (2014) is conceptually appealing but practically ineffective in finding a solution to the regime switching HJB equation for general utility functions, due to the curse of dimensionality, see Remark 2.1 for further discussions on this point.

In this paper we discuss how to derive tight lower and upper bounds of the solution to the regime switching HJB equation. Inspired by the work of Bian et al. (2011) and Bian and Zheng (2015), we extend the dual control approach to regime-switching models. 
The dual value function can be easily computed by the Monte Carlo method as it is an option pricing problem. The dual value function not only provides an upper bound for the primal value function, but also suggests a good feasible control that can be used to find a lower bound for the primal value function. These bounds can be improved, that is, the gap of these bounds can be reduced, with additional controls to the dual process. The suggested method can be applied to general utility functions, including ones not necessarily differentiable and/or strictly concave. The bounds are very tight (less than $1 \%$ of relative error in most of our numerical tests) and may be used as an approximation to the value function. An additional benefit in computing the tight lower bound is that we derive a feedback control which may be used as an approximation to the optimal control. To the best of our knowledge, this is the first time a Monte Carlo algorithm is suggested to find the tight lower and upper bounds of the solution to the regime switching HJB equation for general utility functions. Our numerical results show the method is reliable, effective and accurate for power, non-HARA, and Yaari utilities. The latter two utilities are beyond the scope of any current methods available in finding the value function.

The remaining parts of the paper is arranged as follows. Section 2 introduces the optimal asset allocation problem in regime-switching markets. Section 3 presents the dual control method for finding the tight lower and upper bounds and states the main result (Theorem 3.1) and the Monte Carlo algorithms. Section 4 gives extensive numerical tests for a variety of utility functions, including power, non-HARA and Yaari utilities. Section 5 concludes the paper. Appendix gives the proofs of the solution (8) to stochastic differential equation (7) and Theorem 3.1, and some tables of numerical tests.

\section{Optimal asset allocation in regime-switching models}

In this section, we formulate the stochastic control problem in a regime switching diffusion model. Consider a fixed time horizon $[0, T]$. Let $(\Omega, \mathcal{F}, P)$ be a complete probability space and $W$ a standard Brownian motion and $\boldsymbol{\alpha}$ a continuous time finite state observable Markov chain process $(\mathrm{MCP})$, which are independent of each other, and let $\left\{\mathcal{F}_{t}\right\}$ be the natural filtration generated by $W$ and $\boldsymbol{\alpha}$, completed with all $P$-null sets.

We identify the state space of $\left\{\boldsymbol{\alpha}_{t}\right\}$ as a finite set of unit vectors $\mathbb{E}:=\left\{\mathbf{e}_{1}, \mathbf{e}_{2}, \ldots, \mathbf{e}_{d}\right\}$ where $\mathbf{e}_{i} \in \mathbb{R}^{d}$ is a column vectors with one in the $i$ th position and zeros elsewhere, $i=$ $1, \ldots, d$. Denote by $\mathbf{Q}=\left(q_{i j}\right)_{d \times d}$ the generator of the Markov chain $\left\{\boldsymbol{\alpha}_{t}\right\}$ with $q_{i j} \geq 0$ for $i \neq j$ and $\sum_{j=1}^{d} q_{i j}=0$ for each $i \in \mathbb{D}:=\{1, \ldots, d\}$. The MCP $\boldsymbol{\alpha}$ has a semi-martingale representation

$$
\boldsymbol{\alpha}_{t}=\boldsymbol{\alpha}_{0}+\int_{0}^{t} \mathbf{Q}^{\prime} \boldsymbol{\alpha}_{v} d v+\mathbf{M}_{t}, \quad 0 \leq t \leq T,
$$

where $\mathbf{Q}^{\prime}$ is the transpose of $\mathbf{Q}, \mathbf{M}$ is a purely discontinuous square-integrable martingale with initial value zero, see Elliott et al. (1994).

Assume the financial market consists of one risk-free bond and one risky stock. The bond and stock price processes $B$ and $S$ are assumed to follow the stochastic differential equations (SDEs)

$$
d B_{t}=r_{t} B_{t} d t, \quad d S_{t}=S_{t}\left(\mu_{t} d t+\sigma_{t} d W_{t}\right), \quad 0 \leq t \leq T,
$$

where $r_{t}=\boldsymbol{r} \boldsymbol{\alpha}_{t}, \mu_{t}=\boldsymbol{\mu} \boldsymbol{\alpha}_{t}, \sigma_{t}=\boldsymbol{\sigma} \boldsymbol{\alpha}_{t}$, and $\boldsymbol{r}=\left(r_{1}, \ldots, r_{d}\right)$ is a vector of risk-free interest rates with $r_{i}$ being the rate in regime $i, \boldsymbol{\mu}=\left(\mu_{1}, \ldots, \mu_{d}\right)$ and $\boldsymbol{\sigma}=\left(\sigma_{1}, \ldots, \sigma_{d}\right)$ are vectors of return and volatility rates of the risky asset. Assume all rates are positive constants. 
Denote by $\boldsymbol{\theta}:=\left(\theta_{1}, \ldots, \theta_{d}\right)$ the vector of market price of risk rates with $\theta_{i}=\left(\mu_{i}-r_{i}\right) / \sigma_{i}$ for $i \in \mathbb{D}$.

Let $X$ be the wealth process of a portfolio comprising the bond $B$ and the stock $S$. The wealth process $X$ satisfies the SDE

$$
d X_{t}=X_{t}\left(r_{t} d t+\pi_{t} \sigma_{t}\left(\theta_{t} d t+d W_{t}\right)\right), \quad 0 \leq t \leq T
$$

where $\pi$ is a progressively measurable control process, $\pi_{t}$ represents the proportion of wealth $X_{t}$ invested in risky asset $S_{t}$, and $\theta_{t}=\boldsymbol{\theta} \boldsymbol{\alpha}_{t}$ is the market price of risk at time $t$.

The utility maximization problem is defined by

$$
\sup _{\pi} E\left[U\left(X_{T}\right)\right] \text { subject to (2) }
$$

where $U$ is a utility function that is continuous, increasing and concave on $[0, \infty)$.

Stochastic control is a standard method one may use to solve problem (3). (The other method is convex duality martingale method, see Karatzas and Shreve (1998).) To do so, we define a value function

$$
V_{i}(t, x):=\sup _{\pi \in \Pi_{t}} E_{t, x, i}\left[U\left(X_{T}\right)\right], \quad i \in \mathbb{D},
$$

where $E_{t, x, i}$ is the conditional expectation operator given $X_{t}=x$ and $\boldsymbol{\alpha}_{t}=\mathbf{e}_{i}$ (abbreviated by $\left.\alpha_{t}=i\right)$ for $i \in \mathbb{D}$, and $\Pi_{t}:=\left\{\pi_{s}, s \in[t, T]\right\}$ is the set of all admissible control strategies over $[t, T]$.

It is proved in Fu et al. (2014) and Bäuerle and Rieder (2004) that for a continuous, strictly increasing and strictly concave utility function $U$, the optimal value function $V_{i}$, for $i \in \mathbb{D}$, satisfies the following system of HJB equations

$$
\frac{\partial V_{i}}{\partial t}+r_{i} x \frac{\partial V_{i}}{\partial x}-\frac{1}{2} \theta_{i}^{2}\left(\frac{\partial V_{i}}{\partial x}\right)^{2} / \frac{\partial^{2} V_{i}}{\partial x^{2}}+\sum_{j=1}^{d} q_{i j} V_{j}=0, \quad i \in \mathbb{D}
$$

with boundary condition $V_{i}(T, x)=U(x)$ for $i \in \mathbb{D}$. The verification results are also given in Bäuerle and Rieder (2004) and Fu et al. (2014).

Remark 2.1. When $U$ is a power (or logarithmic) utility, one may write $V_{i}(t, x)=U(x) f_{i}(t)$ (or $\left.V_{i}(t, x)=U(x)+f_{i}(t)\right)$ to reduce (5) to a system of linear ODEs with unknown functions $f_{i}, i \in \mathbb{D}$, which can be solved with matrix exponentials (see Bäuerle and Rieder (2004) and $F u$ et al. (2014)). When $U$ is a general utility, it is difficult to solve (5) as it is a system of fully nonlinear PDEs and, unlike power utility, there are no clues of the solution form. Fu et al. (2014) define a functional operator $\mathcal{M}$ and a sequence of functions $\left\{H_{n}\right\}$ by $H_{n+1}=\mathcal{M} H_{n}$ and show that $H_{n}$ converges to the value function $V$ as $n$ tends to $\infty$. The function $H_{0}$ can be computed with the utility $U$. It also reduces a system of fully coupled nonlinear PDEs to a system of decoupled nonlinear PDEs. This is a conceptually excellent algorithm to find the value function, but it is impractical to implement it for the following reasons: to find $H_{n+1}$, one has to solve a stochastic control problem using the information of $H_{n}$ which can only be derived numerically and the curse of dimensionality quickly arises from the algorithm of Fu et al. (2014).

Remark 2.2. We assume in this paper that the Markov chain $\boldsymbol{\alpha}$ is observable. If $\boldsymbol{\alpha}$ is not observable, for example, when there is no regime switching in volatility process $\sigma_{t}$, i.e., 
$\sigma_{1}=\ldots=\sigma_{d}$, then a filtering problem arises for an investor who observes only the stock prices. Such models with partial information and regime-switching in $\mu$ have been studied in the literature. Honda (2003) introduces the filtered probability as a new state variable and derives the HJB equation with two state variables and reduces the HJB equation to one state variable for power utility. Rieder and Bäuerle (2005) use a similar approach and derive the representation of the value function for power and logarithmic utility. Sass and Haussmann (2004) derive an explicit representation of the optimal trading strategy using HMM (hidden Markov model) filtering results and Malliavin calculus. Elliott et al. (2008) discuss the differences of the models with constant and with switching volatility and explain the reasons why one may use a finite dimensional optimal filter if $\sigma_{t}$ is constant while one has to use moment based regression if $\sigma_{t}$ is a process.

When there is no regime switching, Bian et al. (2011) and Bian and Zheng (2015) apply the dual control method to show that there is a classical solution to the HJB equation and the optimal value function is the conjugate function of the optimal dual value function. Inspired by these works, we will introduce in the following sections an efficient Monte Carlo method based on the dual control framework to find the lower and upper bounds of the optimal value function for general utilities and show that these bounds are tight and can be improved with increased number of the dual control variables. The usefulness of the algorithm is illustrated by numerical tests of power, non-HARA, and Yaari utilities.

\section{Dual-control Monte-Carlo method}

In this section, we assume that the utility function $U$ is continuous, increasing and concave (but not necessarily strictly increasing and concave), and $U(0)=0$. The dual function of $U$ is defined by

$$
\widetilde{U}(y)=\sup _{x \geq 0}(U(x)-x y)
$$

for $y \geq 0$. The function $\widetilde{U}(y)$ is a continuous, decreasing and convex function on $[0, \infty)$ and satisfies $\widetilde{U}(\infty)=0$ (see e.g., Bian and Zheng (2015)). Define a dual process $Y_{t}$

$$
d Y_{t}=Y_{t-}\left(-r_{t} d t-\theta_{t} d W_{t}+C d \mathbf{M}_{t}\right),
$$

where $C$ is a constant row vector in $R^{d}, W$ is the Brownian motion and and $\mathbf{M}_{t}$ is the martingale defined in (1). The solution to (7) at time $T$, with initial condition $Y_{t}=y$, can be written as

$$
Y_{T}=y \exp \left(A_{t, T}\right)
$$

where

$$
A_{t, T}=-\int_{t}^{T}\left(r_{u}+C \mathbf{Q}^{\prime} \boldsymbol{\alpha}_{u}+\frac{1}{2} \theta_{u}^{2}\right) d u-\int_{t}^{T} \theta_{u} d W_{u}+\sum_{t<s \leq T} \ln \left(1+C\left(\boldsymbol{\alpha}_{s}-\boldsymbol{\alpha}_{s-}\right)\right) .
$$

While it is considered well known that $Y_{T}$ in (8) is the solution of SDE (7) at time $T$, the authors do not find a convenient reference that contains the result. A simple proof is given in Appendix A.

The dual value function is defined by

$$
\widetilde{V}_{i}(t, y)=E_{t, y, i}\left[\widetilde{U}\left(Y_{T}\right)\right], \quad i \in \mathbb{D} .
$$


Since $Y$ satisfies a linear $\operatorname{SDE}(7)$ and $\widetilde{U}$ is a decreasing convex function, we know $\widetilde{V}_{i}(t, y)$ is a decreasing convex function for $y>0$ and fixed $t$ and $i$. Denote by $\mathcal{W}_{i}(t, x)$ the dual function of $\widetilde{V}_{i}(t, y)$ for fixed $t$ and $i$, given by

$$
\mathcal{W}_{i}(t, x)=\inf _{y>0}\left[\widetilde{V}_{i}(t, y)+x y\right]
$$

The next theorem is the main result of the paper.

Theorem 3.1. Let $\mathcal{W}_{i}(t, x)$ be given by (11) and $S_{1}$ and $S_{2}$ be sets of vectors $C$ satisfying $\left|C_{i}\right|<1 / 2$ for $i \in \mathbb{D}$. Then the optimal value function $V_{i}(t, x)$ defined in (4) satisfies

$$
V_{i}(t, x) \leq \inf _{C \in S_{1}} \mathcal{W}_{i}(t, x)
$$

Furthermore, let $\widetilde{V}_{i}(t, y)$ be given by (10) and twice continuously differentiable and strictly convex for $y>0$ and fixed $t$ and $i$ and $y=y^{*}$ be the solution of

$$
\frac{\partial \widetilde{V}_{i}(t, y)}{\partial y}+x=0
$$

Let the feedback control $\bar{\pi}_{i}(t, x)$ be defined by

$$
\bar{\pi}_{i}(t, x)=\frac{\theta_{i}}{\sigma_{i}} \frac{y^{*}}{x} \frac{\partial^{2}}{\partial y^{2}} \widetilde{V}_{i}\left(t, y^{*}\right)
$$

for $t \in[0, T]$ and $x>0$ and let $\bar{X}$ be the unique strong solution of SDE (2) with control process $\pi_{t}=\bar{\pi}_{\boldsymbol{\alpha}_{t}}\left(t, \bar{X}_{t}\right)$ and let $\overline{\mathcal{W}}_{i}(t, x)$ be defined by

$$
\overline{\mathcal{W}}_{i}(t, x):=E_{t, x, i}\left[U\left(\bar{X}_{T}\right)\right] .
$$

Then the optimal value function $V_{i}(t, x)$ satisfies

$$
V_{i}(t, x) \geq \sup _{C \in S_{2}} \overline{\mathcal{W}}_{i}(t, x)
$$

Proof. The proof is given in Appendix B.

Remark 3.1. Clearly, if $S_{1} \subset \tilde{S}_{1}$, then

$$
V_{i}(t, x) \leq \inf _{C \in \tilde{S}_{1}} \mathcal{W}_{i}(t, x) \leq \inf _{C \in S_{1}} \mathcal{W}_{i}(t, x)
$$

Using $\tilde{S}_{1}$ instead of $S_{1}$ gives a tighter upper bound but it may be more expensive in computation. The same applies to the lower bound.

Remark 3.2. Next we give some explanation to the choice of control $\bar{\pi}_{i}(t, x)$ for the tight lower bound for $V_{i}(t, x)$. In theory, this is easy as any feasible control $\pi$ and its corresponding wealth process $X$ provide a value $E\left[U\left(X_{T}\right)\right]$, which is a lower bound of $V_{i}(t, x)$. Our objective is to find a lower bound that is as large as possible. When there is no regime switching, Bian et al. (2011) and Bian and Zheng (2015) show that $V_{i}(t, x)=\mathcal{W}_{i}(t, x)$ with $C=0$ and the optimal control is given by

$$
\pi_{i}(t, x)=-\frac{\theta_{i}}{\sigma_{i}} \frac{\frac{\partial}{\partial x} \mathcal{W}_{i}(t, x)}{x \frac{\partial^{2}}{\partial x^{2}} \mathcal{W}_{i}(t, x)},
$$


which can be equivalently computed using the dual value function $\widetilde{V}_{i}(t, y)$ and its derivatives. This suggests strongly to use $\mathcal{W}_{i}(t, x)$ to construct a control which may provide a good lower bound for $V_{i}(t, x)$, even though we only have the relation $V_{i}(t, x) \leq \mathcal{W}_{i}(t, x)$ in the current regime-switching framework. Assume $\widetilde{V}_{i}(t, y)$ is twice continuously differentiable and strictly convex for $y>0$ and fixed $t$ and $i$ and $y=y^{*}$ is the solution of equation (13). (A sufficient condition that ensures the existence of a unique solution to equation (13) is $\lim _{y \rightarrow 0} \frac{\partial \widetilde{V}_{i}(t, y)}{\partial y}=-\infty$ and $\lim _{y \rightarrow \infty} \frac{\partial \widetilde{V}_{i}(t, y)}{\partial y}=0$.) Then we have

$$
\mathcal{W}_{i}(t, x)=\widetilde{V}_{i}\left(t, y^{*}\right)+x y^{*} .
$$

Note that $y^{*}$ is continuously differentiable for $x>0$ and fixed $t$ and $i$ by the implicit function theorem. A simple calculus, using (13), shows that

$$
\frac{\partial}{\partial x} \mathcal{W}_{i}(t, x)=y^{*}, \frac{\partial^{2}}{\partial y^{2}} \widetilde{V}_{i}\left(t, y^{*}\right) \frac{\partial}{\partial x} y^{*}+1=0, \frac{\partial^{2}}{\partial x^{2}} \mathcal{W}_{i}(t, x)=-1 / \frac{\partial^{2}}{\partial y^{2}} \widetilde{V}_{i}\left(t, y^{*}\right) .
$$

Note that $\frac{\partial}{\partial x} y^{*} \neq 0$, otherwise, one would have $1=0$, a contradiction. Substituting the derivatives above into (17), we get a candidate control $\bar{\pi}_{i}(t, x)$ in (14) for the lower bound.

Remark 3.3. The dual control vector $C$ in (7) must satisfy $1+C\left(\boldsymbol{\alpha}_{t}-\boldsymbol{\alpha}_{t-}\right)>0$ for $t \in[0, T]$, see (9). A sufficient condition to ensure this is $\left|C_{i}\right|<1 / 2$ for $i \in \mathbb{D}$. We may also use a general progressively measurable process $\left(C_{t-}\right)_{t \geq 0}$ in (7), provided (9) is well defined, which indicates one may potentially get even tighter lower and upper bounds if $C$ is chosen to be a process, not just a constant vector. When there is no regime switching, $C$ can be set equal to zero.

The Monte-Carlo method can be used to find the tight lower and upper bounds. To compute the tight lower bound, one key step is to find a solution of (13). Assume utility $U$ is strictly concave and satisfies Inada's condition $\left(U^{\prime}(0)=\infty\right.$ and $\left.U^{\prime}(\infty)=0\right)$, which implies that $\widetilde{U}$ is continuously differentiable and $\widetilde{U}(0)=-\infty$ and $\widetilde{U}(\infty)=0$. From (10), the pathwise differentiation method gives

$$
\frac{\partial \widetilde{V}_{i}(t, y)}{\partial y}=\frac{1}{y} E_{t, y, i}\left[Y_{T} \widetilde{U}^{\prime}\left(Y_{T}\right)\right],
$$

which is $-\infty$ for $y$ close to 0 and 0 for $y$ close to $\infty$. Choosing a sufficiently small $y$ and a sufficiently large $y$ such that the expression $\frac{\partial \widetilde{V}_{i}(t, y)}{\partial y}+x$ has opposite signs for $x>0$, we can then use the bisection method to find the solution to equation (13).

The Markov chain process $\boldsymbol{\alpha}$ can be generated in a standard procedure as follows. Assume MCP is at state $i$. Generate two independent standard uniform variables $\zeta_{1}$ and $\zeta_{2}$, define

$$
\tau_{i}=-\frac{1}{q_{i i}} \ln \zeta_{1} .
$$

where $q_{i i}:=\sum_{j \neq i} q_{i j}$ is the intensity rate of MCP jumping from state $i$ to some other state (not decided yet). Then $\tau_{i}$ is the first jump time of MCP from state $i$. To decide which state it jumps to, divide interval $[0,1]$ by $d-1$ subintervals, with length of $q_{i j} / q_{i i}$ for $j \neq i$. Determine subinterval $j$ such that $\zeta_{2}$ falls into it, the MCP has jumped to state $j$ at time $\tau_{i}$. Repeat these steps to generate a sample path of MCP $\boldsymbol{\alpha}$ on the interval $[0, T]$.

The dual control variable $C$ may be chosen randomly by specifying $\left|C_{i}\right|<1 / 2$, although other methods are also possible, such as dividing the interval $(-1 / 2,1 / 2)$ into equally divided subintervals and then choosing all possible combinations of grid points. 
Next we describe the Monte-Carlo method for computing the tight lower and upper bounds at time $t=0$. The tight lower and upper bounds at other time $t$ can be computed similarly. Assume $X_{0}=x, \alpha_{0}=i$ and the dual function $\widetilde{U}$ in (6) are known.

\section{Monte-Carlo method for computing the tight upper bound:}

Step 1: Sample $d$ independent uniform variables $C_{i}$ in $[-0.4,0.4]$, which are components of a vector $C$.

Step 2: Generate $M_{1}$ sample paths of Brownian motion $W$ and MCP $\boldsymbol{\alpha}$, which are used to compute $Y_{T}$ with $Y_{0}=y$ and the average derivatives:

$$
\frac{\partial \widetilde{V}_{i}(0, y)}{\partial y} \approx \frac{1}{y} \frac{1}{M_{1}} \sum_{\ell=1}^{M_{1}} Y_{T} \widetilde{U}^{\prime}\left(Y_{T}\right) .
$$

Step 3: Use the bisection method to solve equation (13) and get the solution $y \approx y^{*}$.

Step 4: Compute the upper bound

$$
\mathcal{W}_{i}(0, x) \approx \tilde{V}_{i}\left(0, y^{*}\right)+x y^{*} .
$$

Step 5: Repeat Steps 1 to $4 N_{1}$ times and then compute the tight upper bound $\inf _{C \in S_{1}} \mathcal{W}_{i}(0, x)$.

\section{Monte-Carlo method for computing the tight lower bound:}

Step 1: Sample $d$ independent uniform variables $C_{i}$ in $[-0.4,0.4]$, which are components of a vector $C$.

Step 2: Generate $M_{2}$ sample paths of Brownian motion $W$ and MCP $\boldsymbol{\alpha}$, which are used to find the control process $\bar{\pi}$ in (14) and the wealth process $\bar{X}$ in (2).

Step 3: Compute the lower bound

$$
\overline{\mathcal{W}}_{i}(0, x) \approx \frac{1}{M_{2}} \sum_{\ell=1}^{M_{2}} U\left(\bar{X}_{T}\right) .
$$

Step 4: Repeat Steps 1 to $3 N_{2}$ times and then compute the tight lower bound $\sup _{C \in S_{2}} \overline{\mathcal{W}}_{i}(0, x)$.

Remark 3.4. It is much more time consuming to compute the tight lower bound than to the tight upper bound. The reason is that one has to generate sample paths of the wealth process $\bar{X}$ and control process $\bar{\pi}$, which requires to solve equation (13) at all grid points of time, not just at $t=0$ as in the case of computing the tight upper bound. However, there is one exception when $U$ is a power utility, the computation time for the tight lower bound is much shorter than that for the tight upper bound, and the tight lower bound with $C=0$ coincides with the primal value, see the example in the next section. 


\section{Case studies for a variety of utility functions}

\subsection{Power utility}

Consider a power utility function:

$$
U(x)=\frac{x^{p}}{p}, \quad 0<p<1
$$

The dual function is given by

$$
\widetilde{U}(y)=\frac{1-p}{p} y^{\frac{p}{p-1}}
$$

Substituting (8), $Y_{T}=y \exp \left(A_{t, T}\right)$, into (10), we have

$$
\widetilde{V}_{i}(t, y)=\frac{1-p}{p} y^{\frac{p}{p-1}} \beta_{i}(t)
$$

where $\beta_{i}(t)=E_{t, i}\left[\exp \left(\frac{p}{p-1} A_{t, T}\right)\right]$. Since $\widetilde{V}_{i}(t, y)$ is $C^{2}$ and strictly convex at $y>0$ and $\frac{\partial}{\partial y} \widetilde{V}_{i}(t, 0)=-\infty$ and $\frac{\partial}{\partial y} \widetilde{V}_{i}(t, \infty)=0$. The conditions of Theorem 3.1 are satisfied and there is a unique solution to equation (13). It follows from definition (11) that

$$
\mathcal{W}_{i}(t, x)=\widetilde{V}_{i}\left(t, y_{i}^{*}\right)+x y_{i}^{*},
$$

where

$$
y_{i}^{*}=\left(\frac{x}{\beta_{i}(t)}\right)^{p-1}
$$

is the solution of $(13)$ with $\widetilde{V}_{i}(t, y)$ given in $(20)$. The tight upper bound on the primal value is given by

$$
\inf _{C_{t}} \mathcal{W}_{i}(t, x)=\inf _{C_{t}}\left\{\frac{1-p}{p}\left(y_{i}^{*}\right)^{\frac{p}{p-1}} \beta_{i}(t)+x y_{i}^{*}\right\}
$$

and can be computed with the Monte-Carlo method.

To calculate the tight lower bound on the primal value, we need first to calculate the control $\bar{\pi}_{i}(t, x)$. A direct computation, using (14), gives that

$$
\bar{\pi}_{i}(t, x)=\frac{\theta_{i}}{\sigma_{i}} \frac{1}{1-p} .
$$

The wealth process becomes

$$
d \bar{X}_{t}=\bar{X}_{t}\left(r_{t} d t+\frac{\theta_{t}}{1-p}\left(\theta_{t} d t+d W_{t}\right)\right)
$$

The solution is given by

$$
\bar{X}_{T}=x \exp \left[\int_{t}^{T}\left(r_{s}+\frac{1-2 p}{2(1-p)^{2}} \theta_{s}^{2}\right) d s+\frac{\theta_{s}}{1-p} d W_{s}\right] .
$$

Since there is a closed-form expression for $\bar{X}_{T}$, we can generate $\bar{X}_{T}$ directly without having to generate a sample path of $\bar{X}$ first. Note also that the control process $\bar{\pi}$ is independent of the dual control vector $C$, which implies the lower bound $\overline{\mathcal{W}}_{i}(t, x)$ is already the tight 
lower bound. These two facts make the computation of the lower bound for power utility very fast, which is not the case for general utilities. The lower bound in (15) is given by

$$
\begin{aligned}
\overline{\mathcal{W}}_{i}(t, x) & =\frac{1}{p} x^{p} E_{t, i}\left[\exp \left[\int_{t}^{T}\left(p r_{s}+\frac{p(1-2 p)}{2(1-p)^{2}} \theta_{s}^{2}\right) d s+\frac{p \theta_{s}}{1-p} d W_{s}\right]\right] \\
& =\frac{1}{p} x^{p} E_{t, i}\left[\exp \left[\int_{t}^{T}\left(p r_{s}+\frac{p}{2(1-p)} \theta_{s}^{2}\right) d s\right]\right] .
\end{aligned}
$$

The expectation after the first equality is over both Brownian motion $W$ and MCP $\boldsymbol{\alpha}$ and the expectation after the second equality is over $\mathrm{MCP} \alpha$ only. We can verify directly that expression (21) satisfies the HJB equation (5) and the terminal condition $\overline{\mathcal{W}}_{i}(T, x)=x^{p} / p$. Therefore, the lower bound $\overline{\mathcal{W}}_{i}(t, x)$ equals the primal value function $V_{i}(t, x)$.

In the next numerical example we use the dual-control Monte-Carlo method to calculate the lower and upper bounds for power utility (19) with $p=1 / 2$. The initial wealth at time $t=0$ is $x=1$, the investment period $T=1$, and the number of simulations is $M_{1}=M_{2}=10^{7}$. Since the terminal wealth $\bar{X}_{T}$ has a closed-form expression, there is no need to use the Euler method to discretize SDE (2) to generate the random variable $\bar{X}_{T}$, which results in the fast computation of the lower bound.

Example 4.1. We consider 2-state Markov chain process with generating matrix

$$
\boldsymbol{Q}=\left(\begin{array}{rr}
-a & a \\
b & -b
\end{array}\right)
$$

where $a, b$ are positive constants. To show the robustness of the algorithm, we have chosen 5 samples of a, $b$ from the uniform distribution on interval $[0.1,2.0]$, which means the transition of one state to another can be slow (average once every 10 years) or fast (average twice a year) or anything in between. The states can be "growth economy" (state 1) and "recession economy" (state 2). The riskless interest rates, return and volatility rates of risky asset are given by

$$
\boldsymbol{r}=(0.05,0.01), \quad \boldsymbol{\mu}=(0.13,0.07), \quad \boldsymbol{\sigma}=(0.20,0.30) .
$$

The comparisons are carried out for the cases of $C=0$ and additional control vector $C$ (sampling $N_{1}=50$ times for computing the tight upper bound and $N_{2}=0$ times for computing the tight lower bound). The reason for choosing $N_{2}=0$ is that the exact lower bound with $C=0$ coincides with the primal value and there is no need to use the additional control $C$ in the computation of the lower bound. In the test, the benchmark value is the primal value explicitly given by (see Bäuerle and Rieder (2004) and Fu et al. (2014))

$$
V(t, x, i)=a(t, i) \frac{x^{p}}{p}, \quad \text { for } t \in[0, T], x \geq 0, i \in \mathbb{D},
$$

where $a(t, i)$ is the ith component of vector $\boldsymbol{a}(t)=\exp [-(\boldsymbol{\Lambda}-\boldsymbol{Q})(T-t)] \cdot \mathbf{1}$ and $\mathbf{1}$ is a $d \times 1$ vector with all components 1 and $\boldsymbol{\Lambda}$ is a $d \times d$ diagonal matrix with diagonal elements $\lambda_{i}=\frac{1}{2} \theta_{i}^{2} \frac{p}{p-1}-p r_{i}$ for $i \in \mathbb{D}$.

The numerics in Table 8 (see Appendix C) show that the dual-control Monte-Carlo method generates correct and tight lower and upper bounds on the primal value and the use of control vector $C$ can decrease further the gap between the lower and upper bounds. We have also listed statistics in Table 8, including the average computation time in seconds, 
the mean and the standard deviation of absolute and relative difference between the lower and upper bounds. The improved upper bound with additional dual control variable $C$ comes with increased computation time. This is a trade-off of accuracy and speed.

In Table 1, we give the mean and standard deviation of the absolute and relative difference between the lower and upper bounds for power utility with many randomly sampled parameters-sets: 100 samples of $a, b$ from the uniform distribution on interval [0.1,2.0], $r_{1}, r_{2}$ on $[0.01,0.08], \mu_{1}, \mu_{2}$ on $[0.03,0.20], \sigma_{1}, \sigma_{2}$ on $[0.1,0.6]$. It is clear that the gap between the tight lower and upper bounds is very small, especially when the dual control $C$ is used. This shows the algorithm is reliable and accurate.

Table 1: Mean and std of the absolute and relative difference between the lower and upper bounds for power utility in Example 4.1 with many randomly sampled parameters-sets.

\begin{tabular}{lcc}
\hline & No Control C & With Control C \\
mean diff & 0.0039 & 0.0009 \\
std diff & 0.0120 & 0.0025 \\
mean rel-diff $(\%)$ & 0.1443 & 0.0328 \\
std rel-diff $(\%)$ & 0.4096 & 0.0837 \\
\hline
\end{tabular}

\subsection{A non-HARA utility}

Consider the following non-HARA utility function from Bian and Zheng (2015):

$$
U(x)=\frac{1}{3} H(x)^{-3}+H(x)^{-1}+x H(x)
$$

for $x>0$, where $H(x)=\sqrt{2}(-1+\sqrt{1+4 x})^{-1 / 2}$. Bian and Zheng (2015) show that $U$ is continuously differentiable, strictly increasing and strictly concave, satisfying $U(0)=0$, $U(\infty)=\infty, U^{\prime}(0)=\infty$ and $U^{\prime}(\infty)=0$. Furthermore, the relative risk aversion coefficient of $U$ is given by

$$
R(x)=-\frac{x U^{\prime \prime}(x)}{U^{\prime}(x)}=\frac{1}{4}\left(1+\frac{1}{\sqrt{1+4 x}}\right),
$$

which shows that $U$ is not a HARA utility and represents an investor who will increase the percentage of wealth invested in the risky asset as wealth increases.

To find the dual function $\widetilde{U}(y)$ we may use the definition (6) to compute the maximum of $U(x)-x y$ over $x>0$. A simple calculus shows that $U^{\prime}(x)=H(x)$, so the maximum is achieved at a point $x^{*}$ satisfying $H\left(x^{*}\right)=y$, which gives $x^{*}=y^{-2}+y^{-4}$. Substituting $x^{*}$ into $U(x)-x y$, we conclude that the dual function is given by

$$
\widetilde{U}(y)=\frac{1}{3} y^{-3}+y^{-1} .
$$

Inserting (8), $Y_{T}=y \exp \left(A_{t, T}\right)$, into (10), we get

$$
\widetilde{V}_{i}(t, y)=E_{t, y, i}\left[\widetilde{U}\left(Y_{T}\right)\right]=\frac{1}{3} y^{-3} D_{t, i}+y^{-1} F_{t, i},
$$

where $D_{t, i}=E_{t, i}\left[\exp \left(-3 A_{t, T}\right)\right]$ and $F_{t, i}=E_{t, i}\left[\exp \left(-A_{t, T}\right)\right]$. It is easy to check that the conditions of Theorem 3.1 are satisfied and there is a unique solution $y^{*}$ to the equation 
(13). From definition (11) we obtain the upper bound

$$
\mathcal{W}_{i}(t, x)=\frac{1}{3}\left(y^{*}\right)^{-3} D_{t, i}+\left(y^{*}\right)^{-1} F_{t, i}+x y^{*},
$$

where

$$
y^{*}=\sqrt{\frac{1}{2 x}\left(F_{t, i}+\sqrt{F_{t, i}^{2}+4 x D_{t, i}}\right)}
$$

is the solution of (13) with $\widetilde{V}_{i}(t, y)$ given by (25). The tight upper bound $\inf _{C \in S_{1}} \mathcal{W}_{i}(t, x)$ can be computed with the Monte-Carlo method.

Now we compute the control $\bar{\pi}_{i}(t, x)$. A direct computation yields, using (14) and (25),

$$
\bar{\pi}_{i}(t, x)=\frac{2 \theta_{i}}{\sigma_{i}} \frac{1}{x}\left[\left(y^{*}\right)^{-4} D_{t, i}+x\right], \quad i \in \mathbb{D} .
$$

Here we have used the relation $\left(y^{*}\right)^{-4} D_{t, i}+\left(y^{*}\right)^{-2} F_{t, i}=x$. The Euler discretization for the wealth process (2) with step size $\Delta t$ is given by

$$
\bar{X}_{t+\Delta t}=\left(1+r_{t} \Delta t\right) \bar{X}_{t}+\bar{X}_{t} \bar{\pi}\left(t, \bar{X}_{t}\right) \sigma_{t}\left(\theta_{t} \Delta t+W_{\Delta t}\right)
$$

with $X_{0}=x$, where $\bar{\pi}\left(t, \bar{X}_{t}\right)=\left(\bar{\pi}_{1}\left(t, \bar{X}_{t}\right), \ldots, \bar{\pi}_{d}\left(t, \bar{X}_{t}\right)\right) \boldsymbol{\alpha}_{t}$. Using the Monte-Carlo method, we can find the tight lower bound

$$
\sup _{C \in S_{2}} \overline{\mathcal{W}}_{i}(t, x)=\sup _{C \in S_{2}} E_{t, x, i}\left[U\left(\bar{X}_{T}\right)\right] .
$$

In the following numerical examples we use the dual-control Monte-Carlo method to calculate the lower and upper bounds for non-HARA utility (24). The initial wealth at time $t=0$ is $x=1$, the investment period $T=1$, and the number of simulations is $M_{1}=M_{2}=10^{6}$. Since there is no closed form expression for the terminal wealth $\bar{X}_{T}$, we use (26) with stepsize $\Delta t=0.01$ to generate sample paths of the wealth process $\bar{X}$ to get $\bar{X}_{T}$, which makes the comptation of the lower bound more expensive (taking longer).

Example 4.2. We compare the lower and upper bounds generated by the dual-control Monte-Carlo with the exact primal values when there is no regime-switching. The primal value function has the following explicit form (see Bian and Zheng (2015)):

$$
V(t, x)=\frac{2}{3}\left(\left(y^{*}\right)^{-1} e^{\left(r+\theta^{2}\right)(T-t)}+2 x y^{*}\right),
$$

where

$$
\left(y^{*}\right)^{2}=\frac{1}{2 x}\left(e^{\left(r+\theta^{2}\right)(T-t)}+\sqrt{e^{2\left(r+\theta^{2}\right)(T-t)}+4 x e^{3\left(r+2 \theta^{2}\right)(T-t)}}\right)
$$

and $\theta=(\mu-r) / \sigma$. By choosing $(r, \mu, \sigma)=(0.05,0.13,0.20)$ and $(0.01,0.07,0.30)$, we can find two exact (benchmark) values of the primal problem. Now we set $\boldsymbol{r}, \boldsymbol{\mu}, \boldsymbol{\sigma}$ as in (23) and the generating matrix $\boldsymbol{Q}$ as in (22) with $a=b=0.005$. Since the probability of a jump of $M C P$ on the interval $[0, T]$ is very small (less than 0.005), we expect to see the lower and upper bounds, starting from initial states 1 and 2 , are close to the exact values for $(r, \mu, \sigma)$ chosen above. Table 2 shows this is indeed the case. 
Table 2: Comparisons of lower and upper bounds with the exact (benchmark) value for Example 4.2 (non-HARA utility).

\begin{tabular}{cccccc}
\hline$\alpha_{0}$ & Benchmark & LB & UB & diff & rel-diff $(\%)$ \\
\hline 1 & 2.6171 & 2.6140 & 2.6172 & 0.0032 & 0.1224 \\
2 & 2.3060 & 2.3059 & 2.3060 & 0.0001 & 0.0034 \\
\hline
\end{tabular}

Table 3: Lower bound (LB) and upper bound (UB) with two-state regime-switching for Example 4.3 (non-HARA utility).

\begin{tabular}{ccccccccc}
\hline & \multicolumn{4}{c}{ No control $C$} & \multicolumn{4}{c}{ With control $C$} \\
$\alpha_{0}$ & LB & UB & diff & rel-diff (\%) & LB & UB & diff & rel-diff (\%) \\
\hline 1 & 2.5569 & 2.5627 & 0.0057 & 0.2243 & 2.5569 & 2.5592 & 0.0022 & 0.0873 \\
2 & 2.3611 & 2.3660 & 0.0049 & 0.2063 & 2.3611 & 2.3622 & 0.0011 & 0.0452 \\
\hline
\end{tabular}

Example 4.3. We now consider a regime switching model with generating matrix $\boldsymbol{Q}$ given by (22) and $a=b=0.5$ and other parameters given by (23). There is no closed-form formula for the exact value. The comparisons are carried out for the cases of $C=0$ and additional control vector $C$ (sampling $N_{1}=50$ times for computing the tight upper bound and $N_{2}=50$ times for computing the tight lower bound). The numerics in Table 3 show that the use of control vector $C$ significantly decreases the difference between the lower and upper bounds.

Using the optimal control vector $C^{*}$ for computing the tight lower bound in Table 3, we draw $2 D$ graphs for sample paths of $M C P \alpha$, feedback control $\bar{\pi}$, optimal wealth process $\bar{X}$ (Figure 1), 3D graphs for feedback control $\bar{\pi}$ (Figure 2), and distributions of the terminal wealth $\bar{X}_{T}$ (Figure 3).

Example 4.4. This numerical test is to show the robustness of the dual control Monte Carlo method. The setup and data used are the same as those in Example 4.1. The comparisons are carried out for the cases of $C=0$ and additional control vector $C\left(N_{1}=50\right.$ and $N_{2}=20$ ). The numerics in Table 9 (see Appendix $C$ ) show that the use of the control vector $C$ significantly decreases the difference between the lower and upper bounds.

In Table 4, we give the mean and standard deviation of the absolute and relative difference between the lower and upper bounds for non-HARA utility with many randomly sampled parameters-sets: 10 samples of $a, b$ from the uniform distribution on interval $[0.1,2.0], r_{1}, r_{2}$ on $[0.01,0.08], \mu_{1}, \mu_{2}$ on $[0.03,0.20], \sigma_{1}, \sigma_{2}$ on $[0.1,0.6]$. It is clear that the gap between the tight lower and upper bounds is very small, especially when the dual control $C$ is used. This shows the algorithm is reliable and accurate.

Example 4.5. We consider 3-state MCP with generating matrix

$$
\boldsymbol{Q}=\left(\begin{array}{rrr}
-1.5 a & a & 0.5 a \\
b & -2 b & b \\
0.5 c & c & -1.5 c
\end{array}\right)
$$

where $a, b, c$ are positive constants. This structure of the generating matrix is to indicate that a state is more likely to move to its adjacent state then to a state farther away. The states can be "growth economy" (state 1), "average economy" (state 2) and "recession economy" 

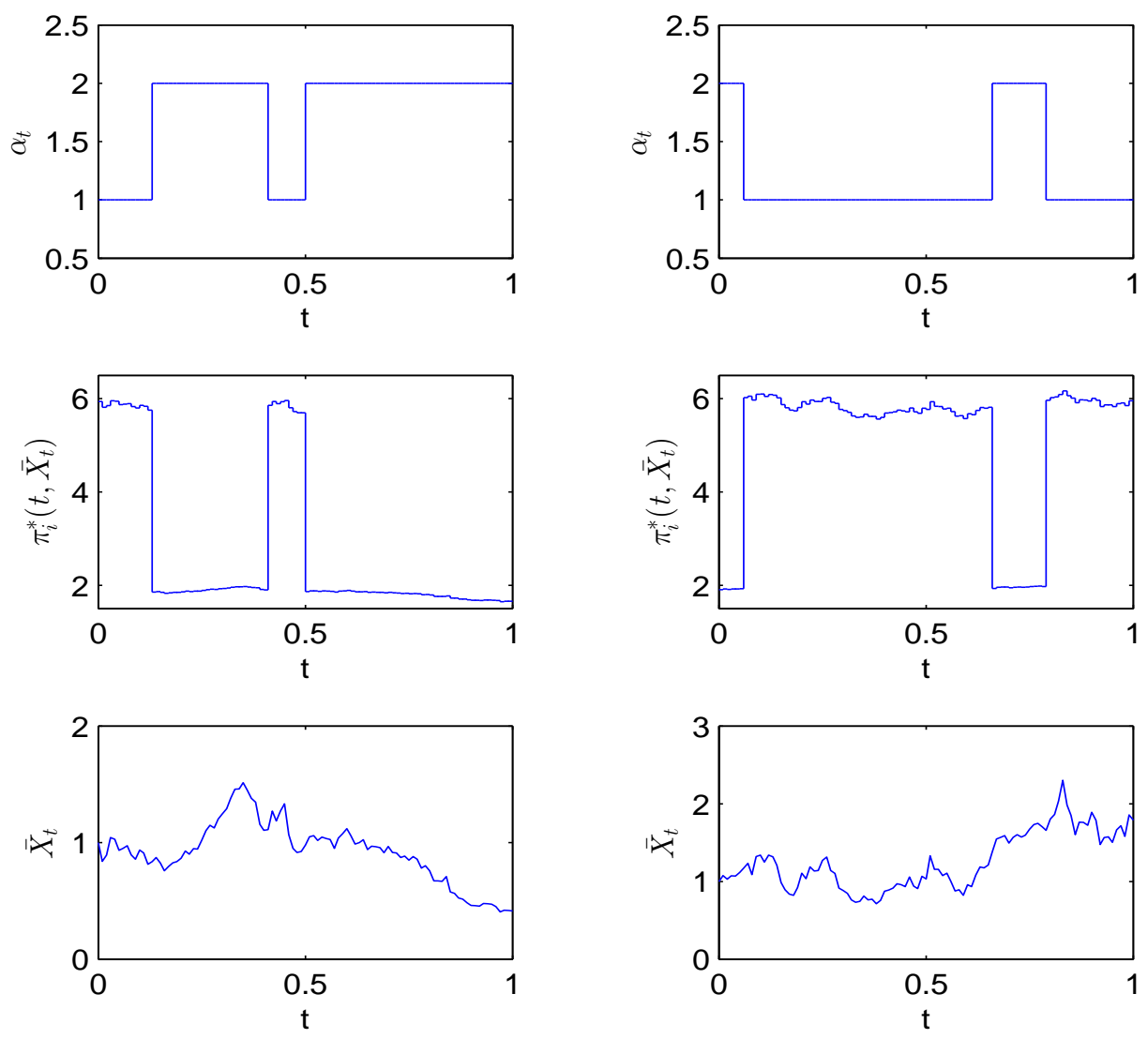

Figure 1: Sample paths of MCP, $\pi\left(t, \bar{X}_{t}\right), \bar{X}_{t}$ with initial wealth $x=1$ (Example 4.3 (non-HARA utility)). The left three figures has the same sample with initial regime state $\alpha_{0}=1$ and optimal control vector $C^{*}=(0.399566,0.310858)$. The right three figures has the same sample with initial regime state $\alpha_{0}=2$ and optimal control vector $C^{*}=$ $(0.023798,-0.028443)$.

Table 4: Mean and std of the absolute and relative difference between the lower and upper bounds for non-HARA utility in Example 4.4 with many randomly sampled parameters-sets.

\begin{tabular}{lcc}
\hline & No Control C & With Control C \\
mean diff & 0.1241 & 0.0184 \\
std diff & 0.3755 & 0.0578 \\
mean rel-diff $(\%)$ & 2.9121 & 0.4885 \\
std rel-diff $(\%)$ & 8.5918 & 1.5245 \\
\hline
\end{tabular}

(state 3). The jump intensities $a, b, c$ are chosen from the uniform distribution on interval $[0.1,2.0]$. The riskless interest rates, return and volatility rates of risky asset are given by

$$
\boldsymbol{r}=(0.06,0.04,0.01), \quad \boldsymbol{\mu}=(0.20,0.12,0.07), \quad \boldsymbol{\sigma}=(0.25,0.20,0.30) .
$$



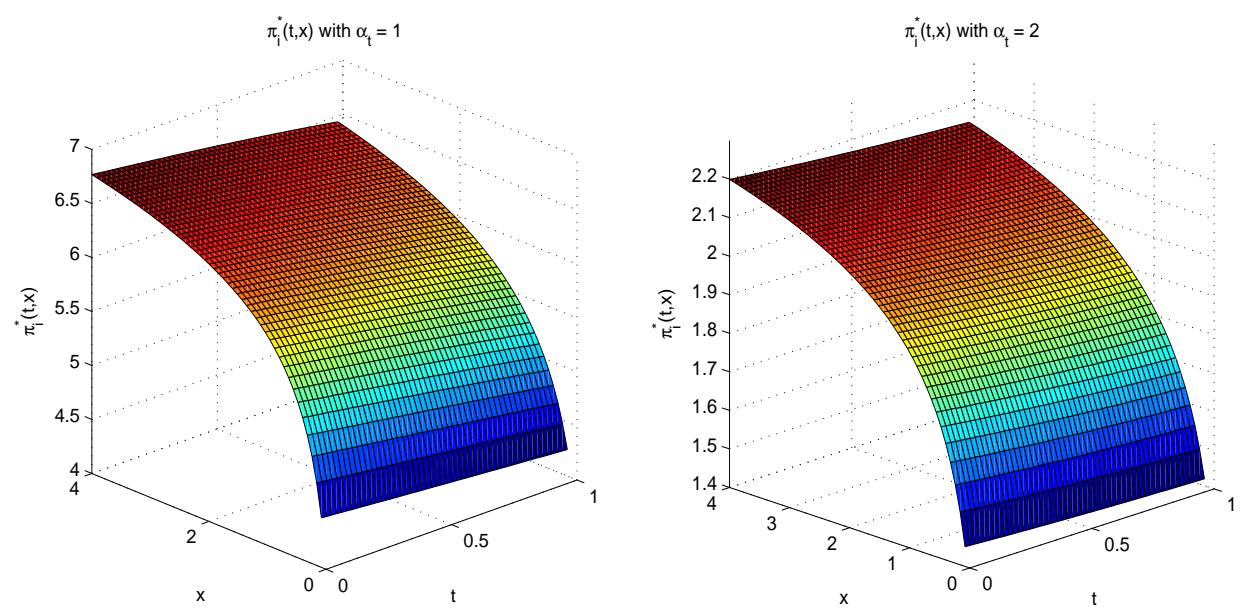

Figure 2: 3D graphs of the optimal control $\pi^{*}(t, x)$ (Example 4.3 (non-HARA utility)). The left figure is for initial regime state $\alpha_{0}=1$ and optimal control vector $C^{*}=(0.399566,0.310858)$ for computing the tight lower bound. The right figure is for initial regime state $\alpha_{0}=2$ and optimal control vector $C^{*}=(0.023798,-0.028443)$ for computing the tight lower bound.

The comparisons are carried out for the cases of $C=0$ and additional control vector $C$ $\left(N_{1}=50\right.$ and $N_{2}=20$ ). The numerics in Table 10 (see Appendix C) show that the use of control vector $C$ significantly decreases the difference between the lower and upper bounds.

\subsection{Yaari utility}

Consider utility function:

$$
U(x)=x \wedge H,
$$

where $H$ is a positive constant. Since the optimal terminal wealth $X_{T}$ is distributed as a Bernoulli distribution similar to the optimal portfolio choice in Yaari dual theory, see Yaari (1987), we call the utility defined in (27) a Yaari utility.

The dual function is given by

$$
\widetilde{U}(y)=H(1-y)^{+},
$$

where $x^{+}=\max (x, 0)$. Inserting (8), $Y_{T}=y \exp \left(A_{t, T}\right)$, into (10), we get the dual value function

$$
\widetilde{V}_{i}(t, y)=E_{t, i}\left[H\left(1-y \exp \left(A_{t, T}\right)\right)^{+}\right],
$$

where the expectation is over Brownian motion $W$ and MCP $\boldsymbol{\alpha}$. Given $\boldsymbol{\alpha}, A_{t, T}$ is a normal variable with mean $m_{t, T}$ and variance $\sigma_{t, T}^{2}$, given by

$$
\begin{aligned}
m_{t, T} & =-\int_{t}^{T}\left(r_{u}+C \mathbf{Q}^{\prime} \boldsymbol{\alpha}_{u}+\frac{1}{2} \theta_{u}^{2}\right) d u+\sum_{t<s \leq T} \ln \left(1+C\left(\boldsymbol{\alpha}_{s}-\boldsymbol{\alpha}_{s-}\right)\right), \\
\sigma_{t, T}^{2} & =\int_{t}^{T} \theta_{u}^{2} d u .
\end{aligned}
$$



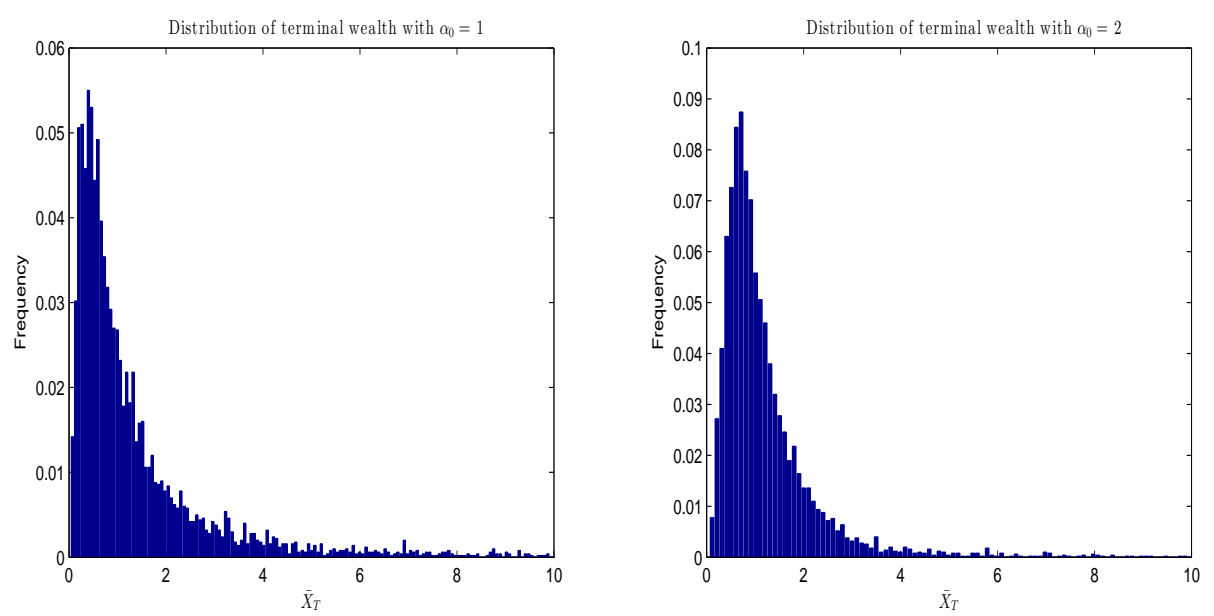

Figure 3: Distribution of terminal wealth process for non-HARA utility (Example 4.3).

The dual value function $\widetilde{V}_{i}(t, y)$ is simply a European put option price, for a given MCP $\boldsymbol{\alpha}$, and can be simplified further as

$\widetilde{V}_{i}(t, y)=E_{t, i}\left[H \Phi\left(\frac{-\ln y-m_{t, T}}{\sigma_{t, T}}\right)-H y \exp \left(m_{t, T}+\frac{1}{2} \sigma_{t, T}^{2}\right) \Phi\left(\frac{-\ln y-m_{t, T}}{\sigma_{t, T}}-\sigma_{t, T}\right)\right]$,

where the expectation is over MCP $\boldsymbol{\alpha}$ only and $\Phi$ is the cumulative distribution function of a standard normal variable. The derivatives of $\widetilde{V}_{i}(t, y)$ are given by

$$
\begin{aligned}
\frac{\partial \widetilde{V}_{i}(t, y)}{\partial y} & =-E_{t, i}\left[H \exp \left(m_{t, T}+\frac{1}{2} \sigma_{t, T}^{2}\right) \Phi\left(\frac{-\ln y-m_{t, T}}{\sigma_{t, T}}-\sigma_{t, T}\right)\right], \\
\frac{\partial^{2} \widetilde{V}_{i}(t, y)}{\partial y^{2}} & =E_{t, i}\left[\frac{H}{y \sigma_{t, T}} \exp \left(m_{t, T}+\frac{1}{2} \sigma_{t, T}^{2}\right) \phi\left(\frac{-\ln y-m_{t, T}}{\sigma_{t, T}}-\sigma_{t, T}\right)\right] .
\end{aligned}
$$

Using definition (11), we obtain the upper bound

$$
\mathcal{W}_{i}(t, x)=\widetilde{V}_{i}\left(t, y^{*}\right)+x y^{*},
$$

where $y^{*}$ is the solution of (13) with $\frac{\partial \widetilde{V}_{i}(t, y)}{\partial y}$ given by (29), i.e.,

$$
-E_{t, i}\left[H \exp \left(m_{t, T}+\frac{1}{2} \sigma_{t, T}^{2}\right) \Phi\left(\frac{-\ln y-m_{t, T}}{\sigma_{t, T}}-\sigma_{t, T}\right)\right]+x=0 .
$$

There is no explicit formula for the solution to equation (30). The bisection method can be applied to solve equation (30). In addition, the left-hand side of (30) is increasing with $y$, thus the equation (30) has an unique root if and only if the left-hand side of (30) is less than zero when $y$ goes to zero and greater than 0 when $y$ goes to positive infinity. This gives a condition to ensure equation (30) has a unique root, i.e.,

$$
x<E_{t, i}\left[H \exp \left(m_{t, T}+\frac{1}{2} \sigma_{t, T}^{2}\right)\right] .
$$

If (31) is not satisfied, then $\widetilde{V}_{i}(t, y)+x y$ is an increasing function of $y>0$, which implies the minimum in the definition of $\mathcal{W}_{i}(t, x)$, see $(11)$, is achieved at $y^{*}=0$. Therefore, $\mathcal{W}_{i}(t, x)=H$, an obvious upper bound as $U(x)=x \wedge H \leq H$ for all $x>0$. 
The tight upper bound $\inf _{C \in S_{1}} \mathcal{W}_{i}(t, x)$ can be computed with the Monte-Carlo method. The control $\bar{\pi}_{i}(t, x)$ can be computed by the following formula:

(i) When condition (31) holds, we use (14) to compute $\bar{\pi}_{i}(t, x)$ and get

$$
\bar{\pi}_{i}(t, x)=\frac{\theta_{i}}{\sigma_{i}} \frac{y_{i}^{*}}{x} E_{t, i}\left[\frac{H}{y^{*} \sigma_{t, T}} \exp \left(m_{t, T}+\frac{1}{2} \sigma_{t, T}^{2}\right) \phi\left(\frac{-\ln y^{*}-m_{t, T}}{\sigma_{t, T}}-\sigma_{t, T}\right)\right] .
$$

(ii) When constraint (31) is not satisfied, the best strategy is to invest all the money into the risk-free asset, i.e.,

$$
\bar{\pi}_{i}(t, x)=0 .
$$

We can then generate sample paths of the wealth process $\bar{X}$ with the Euler method as in (26) and find the tight lower bound $\sup _{C \in S_{2}} \overline{\mathcal{W}}_{i}(t, x)$ with the Monte Carlo method.

In the following numerical examples we use the dual-control Monte-Carlo method to calculate the lower and upper bounds for Yaari utility. The data used are: the initial wealth $x=1$ at time $t=0$, the investment period $T=1$ and the threshold level $H=2$. Since there is no closed form expression for terminal wealth $\bar{X}_{T}$, we use (26) with stepsize $\Delta t=0.02$ to generate sample paths of the wealth process $\bar{X}$ to get $\bar{X}_{T}$. There is a further complication in computing the lower bound as we have to find the solution $y^{*}$ of equation (30) numerically. We use the bisection method with the error tolerance $10^{-6}$. It is time consuming to compute the lower bound, so we only choose $M_{1}=M_{2}=5000$ simulations.

Example 4.6. We compare the lower and upper bounds generated by the dual-control Monte-Carlo with the exact primal values when there is no regime-switching. The primal value function has the following explicit form (see Bian and Zheng (2015)):

$$
V(t, x)= \begin{cases}H \Phi\left(\Phi^{-1}\left(\frac{x}{H} e^{r(T-t)}\right)+\theta \sqrt{T-t}\right), & 0 \leq x<H e^{-r(T-t)}, \\ H, & x \geq H e^{-r(T-t)},\end{cases}
$$

where $\theta=(\mu-r) / \sigma$ is a constant. The other data used are the same as those in Example 4.2. The numerical results in Table 5 show that the lower and upper bounds coincide with the exact value ignoring the Monte-Carlo simulation errors.

Table 5: Comparisons of lower and upper bounds with the exact (benchmark) value for Example 4.6 (Yaari utility).

\begin{tabular}{cccccc}
\hline$\alpha_{0}$ & Benchmark & LB & UB & diff & rel-diff (\%) \\
\hline 1 & 1.3576 & 1.3374 & 1.3576 & 0.0202 & 1.5072 \\
2 & 1.1684 & 1.1569 & 1.1684 & 0.0114 & 0.9888 \\
\hline
\end{tabular}

Example 4.7. The setup and data used in this numerical test are the same as those in Example 4.3. The comparisons are carried out for the cases of $C=0$ and additional control vector $C\left(N_{1}=100\right.$ and $\left.N_{2}=10\right)$. The numerics in Table 6 show that the use of the control vector $C$ significantly decreases the difference between the lower and upper bounds.

Using the optimal control vector $C^{*}$ for computing the tight lower bound in Table 6, we plot $3 D$ graphs for the optimal control $\pi^{*}(t, x)$ (Figure 4) and distributions of the terminal wealth $\bar{X}_{T}$ (Figure 5). 
Table 6: Lower bound (LB) and upper bound (UB) with two-state regime-switching for Example 4.7 (Yaari utility).

\begin{tabular}{ccccccccc}
\hline \multicolumn{4}{c}{ No control $C$} & \multicolumn{4}{c}{ With control $C$} \\
$\alpha_{0}$ & LB & UB & diff & rel-diff $(\%)$ & LB & UB & diff & rel-diff (\%) \\
\hline 1 & 1.3001 & 1.3270 & 0.0269 & 2.0677 & 1.3069 & 1.3267 & 0.0199 & 1.5217 \\
2 & 1.1721 & 1.2070 & 0.0349 & 2.9748 & 1.1815 & 1.2069 & 0.0254 & 2.1465 \\
\hline
\end{tabular}
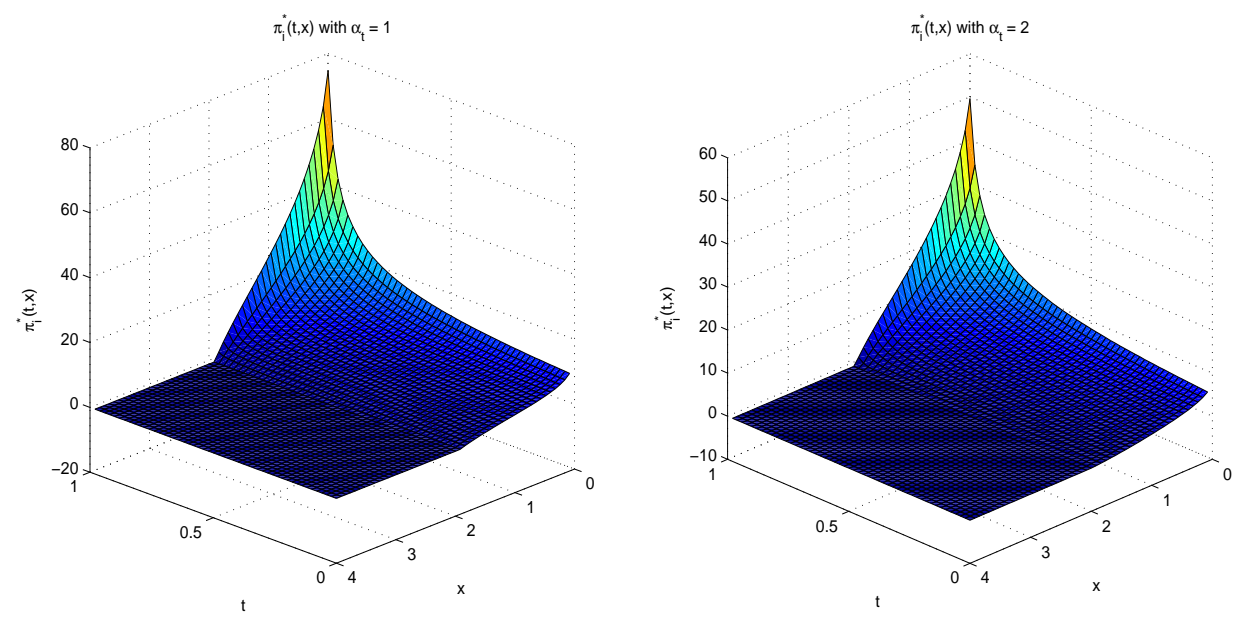

Figure 4: 3D graphs of the optimal control $\pi^{*}(t, x)$ (Example 4.7 (Yaari utility)). The left figure is for initial regime state $\alpha_{t}=1$ and optimal control vector $C^{*}=(0.070462,0.261362)$ for computing the tight lower bound. The right figure is for initial regime state $\alpha_{t}=2$ and optimal control vector $C^{*}=(-0.093885,-0.332256)$ for computing the tight lower bound.

Example 4.8. The setup and data used in this numerical test are the same as those in Example 4.1. The comparisons are carried out for the cases of $C=0$ and additional control vector $C\left(N_{1}=100\right.$ and $\left.N_{2}=5\right)$. The numerics in Table 11 (see Appendix $C$ ) show that the use of the control vector $C$ significantly decreases the difference between the lower and upper bounds.

In Table 7, we give the mean and standard deviation of the absolute and relative difference between the lower and upper bounds for Yaari utility with many randomly sampled parameters-sets: 10 samples of $a, b$ from the uniform distribution on interval $[0.1,2.0], r_{1}, r_{2}$ on $[0.01,0.08], \mu_{1}, \mu_{2}$ on $[0.03,0.20], \sigma_{1}, \sigma_{2}$ on $[0.1,0.6]$. It is clear that the gap between the tight lower and upper bounds is small, but the effect of the dual control $C$ is less pronounced than in the cases for power and non-HARA utilities. The algorithm is still reliable and accurate even for non-differentiable and non-strictly-concave utilities.

\section{Conclusions}

In this paper, we study the dual control approach for the optimal asset allocation problem in a continuous-time regime-switching market. We find the tight lower and upper bounds of the value function that is a solution to the HJB equation, a system of fully coupled nonlinear 

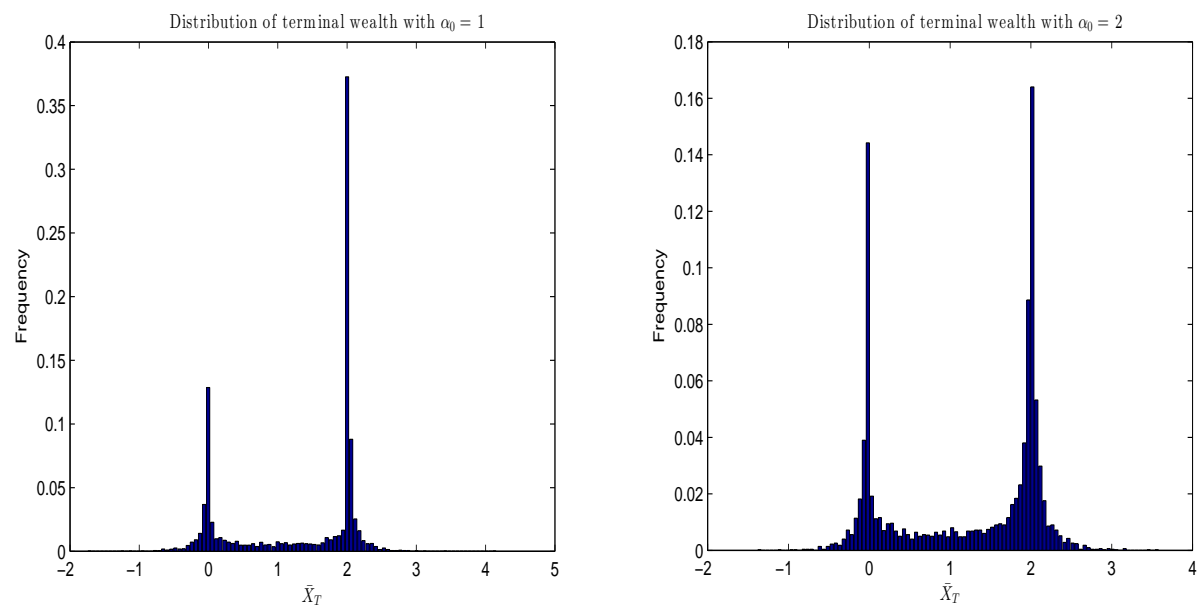

Figure 5: Distribution of terminal wealth process for Yaari utility (Example 4.7).

Table 7: Mean and std of the absolute and relative difference between the lower and upper bounds for Yaari utility in Example 4.8 with many randomly sampled parameters-sets.

\begin{tabular}{lcc}
\hline & No Control C & With Control C \\
mean diff & 0.0345 & 0.0276 \\
std diff & 0.0219 & 0.0122 \\
mean rel-diff (\%) & 2.7910 & 2.2435 \\
std rel-diff $(\%)$ & 1.5750 & 0.8950 \\
\hline
\end{tabular}

partial differential equations. We suggest a Monte-Carlo algorithm for computing these tight lower and upper bounds and show the method is reliable and accurate with a number of numerical tests for power, non-HARA and Yaari utility functions. We can therefore find the approximate value function and its corresponding control strategies numerically for general utility functions in a regime switching Black-Scholes model.

\section{Acknowledgements}

The authors are grateful to the anonymous reviewers whose constructive comments and suggestions have helped to improve the paper of the previous version.

\section{References}

Bäuerle, N. and Rieder, U. (2004). Portfolio optimization with Markov-modulated stock prices and interest rates, IEEE Transactions on Automatic Control, 29, 442-447.

Bian, B., Miao, S. and Zheng, H. (2011). Smooth value functions for a class of nonsmooth utility maximization problems, SIAM Journal of Financial Mathematics, 2, 727-747.

Bian, B. and Zheng, H. (2015). Turnpike property and convergence rate for an investment model with general utility functions, Journal of Economic Dynamics and Control, 51, $28-49$. 
Çanakoğlu, E. and Özekici, S. (2012). HARA frontiers of optimal portfolios in stochastic markets, European Journal of Operational Research, 221, 129-137.

Çelikyurt, U. and Özekici, S. (2007). Multiperiod portfolio optimization models in stochastic markets using the mean-variance approach, European Journal of Operational Research, $179,186-202$.

Elliott, R., Aggoun, L. and Moore, J. (1994). Hidden Markov Models: Estimation and Control, Springer.

Elliott, R., Krishnamurthy, V. and Sass, J. (2008). Moment based regression algorithms for drift and volatility estimation in continuous-time Markov switching models, Econometrics Journal, 11, 244-270.

Forsyth, P.A. and Labahn, G. (2008). Numerical methods for controlled Hamilton-JacobiBellman PDEs in finance, Journal of Computational Finance, 11, 1-44.

Fu, J., Wei, J. and Yang, H. (2014). Portfolio optimization in a regime-switching market with derivatives, European Journal of Operational Research, 233, 184-192.

Glasserman, P. (2004). Monte Carlo Methods in Financial Engineering, Springer.

Hamilton, J.D. (1989). A new approach to the economic analysis of nonstationary time series and the business cycle, Ecomometrica, 57, 357-384.

Hardy, M.R. (2001). A regime-switching model for long-term stock returns, North American Actuarial Journal, 5, 41-53.

Honda, T. (2003). Optimal portfolio choice for unobservable and regime-switching mean returns, Journal of Economic Dynamics \& Control, 28, 45-78.

Huang, Y., Forsyth, P.A. and Labahn, G. (2012). Combined fixed point and policy iteration for HJB equations in finance, SIAM Journal on Numerical Analysis, 50, 1849-1860.

Huang, Y., Forsyth, P.A. and Labahn, G. (2011). Methods for pricing American options under regime switching, SIAM Journal on Scientific Computing, 33, 2144-2168.

Karatzas, I. and Shreve, S.E. (1998). Methods of Mathematical Finance, Springer.

Pham, H. (2009). Continuous-Time Stochastic Control and Optimization with Financial Applications, Springer.

Protter, P.E. (2005). Stochastic Integration and Differential Equations, Second Edition, Springer.

Reisinger, C. and Forsyth, P.A. (2016). Piecewise constant policy approximations to Hamilton-Jacobi-Bellman equations, Applied Numerical Mathematics, 103, 27-47.

Rieder U. and Bäuerle, N. (2005). Portfolio optimization with unobservable Markovmodulated drift process, Journal of Applied Probability, 43, 362-378.

Sass, J. and Haussmann, U.G. (2004). Optimizing the terminal wealth under partial information: The drift process as a continuous time Markov chain, Finance and Stochastics, $8,553-577$. 
Yaari, M.E. (1987). The dual theory of choice under risk, Econometrica, 55, 95-115.

Yao, D.D., Zhang, Q. and Zhou, X.Y. (2006). A regime-switching model for European options, in Stochastic Processes, Optimization, and Control Theory: Applications in Financial Engineering, Queueing Networks, and Manufacturing Systems, (H.M. Yan, G. Yin, and Q. Zhang Eds.), Springer, pp. 281-300.

Yin, G., Zhang, Q., Liu, F., Liu, R.H., and Cheng, Y. (2006). Stock liquidation via stochastic approximation using NASDAQ daily and intra-day data, Mathematical Finance, 16, 217236.

Zhang, Q., Yin, G. and Liu, R.H. (2005). A near-optimal selling rule for a two-time-scale market model, SIAM Journal of Multiscale Modeling \& Simulation, 4, 172-193.

Zhou X.Y. and Yin, G. (2003). Markowitz's mean-variance portfolio selection with regime switching: a continuous-time model, SIAM Journal on Control and Optimization, 42, $1466-1482$.

\section{Appendix A: Proof of (8)}

We verify $Y_{T}$ defined in (8) is the solution of SDE (7) at time $T$. Define

$$
\begin{aligned}
X_{s}^{c} & =-\int_{t}^{s}\left(r_{u}+C \mathbf{Q}^{\prime} \boldsymbol{\alpha}_{u}+\frac{1}{2} \theta_{u}^{2}\right) d u-\int_{t}^{s} \theta_{u} d W_{u}, \\
X_{s}^{j} & =\sum_{t<u \leq s} \ln \left(1+C\left(\boldsymbol{\alpha}_{u}-\boldsymbol{\alpha}_{u-}\right)\right),
\end{aligned}
$$

for $t \leq s \leq T$. Then $X_{s}^{c}$ is a continuous process and $X_{s}^{j}$ is a pure jump process. Define $X_{s}=X_{s}^{c}+X_{s}^{j}$ and $Y_{s}=f\left(X_{s}\right)$ for $t \leq s \leq T$ with $f(x)=y \exp (x)$. Using Ito's formula for semi-martingale processes, see Protter (2005), we have

$$
d Y_{s}=Y_{s} d X_{s}^{c}+\frac{1}{2} Y_{s} d\left[X^{c}, X^{c}\right]_{s}+Y_{s}-Y_{s-},
$$

for $t<s \leq T$, where $\left[X^{c}, X^{c}\right]_{s}=\int_{0}^{s} \theta_{u}^{2} d u$ is the quadratic variation of $X^{c}$ at $s$ and $Y_{s}-Y_{s-}$ is the pure jump part of $Y$ at $s$. Since

$$
Y_{s}=Y_{s-} \exp \left(\ln \left(1+C\left(\boldsymbol{\alpha}_{s}-\boldsymbol{\alpha}_{s-}\right)\right)\right)=Y_{s-}\left(1+C\left(\boldsymbol{\alpha}_{s}-\boldsymbol{\alpha}_{s-}\right)\right)
$$

we have

$$
Y_{s}-Y_{s-}=Y_{s-} C\left(\boldsymbol{\alpha}_{s}-\boldsymbol{\alpha}_{s-}\right) .
$$

Substituting $\left[X^{c}, X^{c}\right]_{s}$ and $Y_{s}-Y_{s-}$ into $d Y_{s}$, also noting (1), we derive SDE (7). Therefore, $Y_{T}$ is the solution of (7) at time $T$. 


\section{Appendix B: Proof of Theorem 3.1}

Using Ito's formula, we have

$$
d\left(X_{t} Y_{t}\right)=X_{t} Y_{t}\left(-\theta_{t}+\pi_{t} \sigma_{t}\right) d W_{t}+X_{t} Y_{t} C d \mathbf{M}_{t},
$$

which implies that the process $X Y$ is a local-martingale and therefore a super-martingale due to $X_{t} Y_{t}$ being non-negative. This, together with (6), gives the following relation

$$
E_{t, x, i}\left[U\left(X_{T}\right)\right] \leq E_{t, y, i}\left[\widetilde{U}\left(Y_{T}\right)\right]+x y .
$$

Since the left side of (32) is independent of $y$ whereas the right side of (32) is independent of $\pi$, we have, see (4),

$$
V_{i}(t, x) \leq \mathcal{W}_{i}(t, x)
$$

where $\mathcal{W}_{i}(t, x)$ is defined by $(11)$. Note that $\mathcal{W}_{i}(t, x)$ depends on $C$. If $S_{1}$ is a set of vectors $C$, then we must have (12) from (33), which gives the tight upper bound.

The tight lower bound (16) is obvious as the process $\pi_{t}=\bar{\pi}_{\boldsymbol{\alpha}_{t}}\left(t, \bar{X}_{t}\right), 0 \leq t \leq T$, is an admissible control process by the assumption of the theorem and $V_{i}(t, x)$ is the optimal value function.

\section{Appendix C: Tables 8, 9, 10, 11}

We list the tables for Examples 4.1, 4.4, 4.5, and 4.8 with random choices of generator matrix $\mathbf{Q}$.

Table 8: Numerical results for Example 4.1 (power utility) with random choices of generator matrix $\mathbf{Q}$ for two-state regime switching.

\begin{tabular}{ccccccc}
\hline & & & \multicolumn{2}{c}{ No Control C } & \multicolumn{2}{c}{ With Control C } \\
$a, b$ & $\alpha_{0}$ & Benchmark & LB & UB & LB & UB \\
\hline 0.100015 & 1 & 2.2139 & 2.2139 & 2.2141 & 2.2139 & 2.2140 \\
0.349922 & 2 & 2.0762 & 2.0761 & 2.0767 & 2.0761 & 2.0764 \\
\hline 1.535650 & 1 & 2.1542 & 2.1537 & 2.1549 & 2.1537 & 2.1545 \\
0.971435 & 2 & 2.0918 & 2.0918 & 2.0924 & 2.0918 & 2.0921 \\
\hline 1.112258 & 1 & 2.1616 & 2.1615 & 2.1623 & 2.1615 & 2.1620 \\
0.516022 & 2 & 2.0776 & 2.0774 & 2.0781 & 2.0774 & 2.0778 \\
\hline 0.189385 & 1 & 2.2110 & 2.2110 & 2.2113 & 2.2110 & 2.2112 \\
1.389843 & 2 & 2.1245 & 2.1243 & 2.1253 & 2.1243 & 2.1249 \\
\hline 1.390663 & 1 & 2.1692 & 2.1690 & 2.1698 & 2.1690 & 2.1694 \\
1.875917 & 2 & 2.1188 & 2.1188 & 2.1194 & 2.1188 & 2.1190 \\
\hline & & ave time & 4.67 & 4.63 & 4.59 & 228.83 \\
& & diff & rel-diff $(\%)$ & diff & rel-diff $(\%)$ \\
& & mean & 0.0007 & 0.0322 & 0.0004 & 0.0181 \\
& std & 0.0003 & 0.0139 & 0.0002 & 0.0095 \\
\hline
\end{tabular}


Table 9: Numerical results for Example 4.4 (non-HARA utility) with random choices of generator matrix $\mathbf{Q}$ for two-state regime switching.

\begin{tabular}{cccccc}
\hline & & \multicolumn{2}{c}{ No Control C } & \multicolumn{2}{c}{ With Control C } \\
$a, b$ & $\alpha_{0}$ & LB & UB & LB & UB \\
\hline 0.100015 & 1 & 2.6021 & 2.6049 & 2.6021 & 2.6040 \\
0.349922 & 2 & 2.3510 & 2.3560 & 2.3510 & 2.3525 \\
\hline 1.535650 & 1 & 2.4925 & 2.4981 & 2.4925 & 2.4934 \\
0.971435 & 2 & 2.3785 & 2.3836 & 2.3785 & 2.3801 \\
\hline 1.112258 & 1 & 2.5060 & 2.5125 & 2.5061 & 2.5073 \\
0.516022 & 2 & 2.3539 & 2.3580 & 2.3539 & 2.3548 \\
\hline 0.189385 & 1 & 2.5967 & 2.5996 & 2.5967 & 2.5984 \\
1.389843 & 2 & 2.4368 & 2.4450 & 2.4368 & 2.4394 \\
\hline 1.390663 & 1 & 2.5188 & 2.5244 & 2.5188 & 2.5215 \\
1.875917 & 2 & 2.4264 & 2.4324 & 2.4264 & 2.4290 \\
\hline & ave time & 102.08 & 0.49 & 2063.39 & 24.55 \\
& & diff & rel-diff $(\%)$ & diff & rel-diff $(\%)$ \\
& mean & 0.0052 & 0.2115 & 0.0018 & 0.0719 \\
& std & 0.0016 & 0.0673 & 0.0007 & 0.0266 \\
\hline
\end{tabular}

Table 10: Numerical results for Example 4.5 (non-HARA utility) with random choices of generator matrix $\mathbf{Q}$ for three-state regime switching.

\begin{tabular}{cccccc}
\hline & & \multicolumn{2}{c}{ No Control C } & \multicolumn{2}{c}{ With Control C } \\
$a, b, c$ & $\alpha_{0}$ & LB & UB & LB & UB \\
\hline 0.100015 & 1 & 3.0018 & 3.0170 & 3.0018 & 3.0134 \\
0.349922 & 2 & 2.6440 & 2.6561 & 2.6440 & 2.6479 \\
1.535650 & 3 & 2.5688 & 2.5973 & 2.5688 & 2.5780 \\
\hline 0.971435 & 1 & 2.7853 & 2.8192 & 2.7853 & 2.8009 \\
1.112258 & 2 & 2.6005 & 2.6254 & 2.6005 & 2.6124 \\
0.516022 & 3 & 2.4137 & 2.4322 & 2.4137 & 2.4219 \\
\hline 0.189385 & 1 & 2.9749 & 2.9954 & 2.9749 & 2.9897 \\
1.389843 & 2 & 2.7014 & 2.7280 & 2.7014 & 2.7095 \\
1.390663 & 3 & 2.5641 & 2.5999 & 2.5641 & 2.5791 \\
\hline 1.875917 & 1 & 2.6998 & 2.7249 & 2.6998 & 2.7155 \\
0.828654 & 2 & 2.5946 & 2.6091 & 2.5946 & 2.6057 \\
1.086891 & 3 & 2.4688 & 2.4887 & 2.4693 & 2.4811 \\
\hline 1.678834 & 1 & 2.6913 & 2.7160 & 2.6940 & 2.7009 \\
0.165687 & 2 & 2.5915 & 2.5961 & 2.5915 & 2.5955 \\
0.201577 & 3 & 2.3509 & 2.3575 & 2.3509 & 2.3535 \\
\hline & ave time & 165.08 & 0.60 & 3273.52 & 29.76 \\
& & diff & rel-diff (\%) & diff & rel-diff $(\%)$ \\
& mean & 0.0208 & 0.7836 & 0.0100 & 0.3750 \\
& std & 0.0091 & 0.3427 & 0.0044 & 0.1570 \\
\hline
\end{tabular}


Table 11: Numerical results for Example 4.8 (Yaari utility) with random choices of generator matrix $\mathbf{Q}$ for two-state regime switching.

\begin{tabular}{cccccc}
\hline & \multicolumn{2}{c}{ No Control C } & \multicolumn{2}{c}{ With Control C } \\
$a, b$ & $\alpha_{0}$ & LB & UB & LB & UB \\
\hline 0.100015 & 1 & 1.3242 & 1.3506 & 1.3308 & 1.3506 \\
0.349922 & 2 & 1.1629 & 1.1998 & 1.1763 & 1.1997 \\
\hline 1.535650 & 1 & 1.2713 & 1.2923 & 1.2713 & 1.2922 \\
0.971435 & 2 & 1.1911 & 1.2205 & 1.1951 & 1.2200 \\
\hline 1.112258 & 1 & 1.2637 & 1.2984 & 1.2723 & 1.2981 \\
0.516022 & 2 & 1.1683 & 1.2027 & 1.1831 & 1.2026 \\
\hline 0.189385 & 1 & 1.3265 & 1.3481 & 1.3265 & 1.3481 \\
1.389843 & 2 & 1.2371 & 1.2595 & 1.2371 & 1.2594 \\
\hline 1.390663 & 1 & 1.2827 & 1.3088 & 1.2906 & 1.3087 \\
1.875917 & 2 & 1.2147 & 1.2530 & 1.2319 & 1.2526 \\
\hline & ave time & 3218.12 & 0.02 & 16515.24 & 1.60 \\
& & diff & rel-diff $(\%)$ & diff & rel-diff $(\%)$ \\
& mean & 0.0291 & 2.3617 & 0.0217 & 1.7394 \\
& std & 0.0066 & 0.6142 & 0.0024 & 0.2325 \\
\hline
\end{tabular}

